\title{
Celiac Artery Aneurysm: Ultrasonic Diagnosis
}

\author{
Gary M. Herzler, MD, Terry M. Silver, MD, Linda M. Graham, MD,
} and James C. Stanley, MD

Celiac artery aneurysms represent an important, although unusual, form of splanchnic vascular disease. Since rupture is a significant complication, surgical intervention is recommended following diagnosis. ${ }^{1}$ Inasmuch as most celiac aneurysms are asymptomatic or present with vague epigastric pain prior to rupture, preoperative diagnosis is infrequent and is usually made incidentally during unrelated surgery or angiography. To our knowledge, a case in which routine abdominal sonography provided the correct diagnosis initially has not been reported.

\section{CASE REPORT}

A 68-yr-old white woman presented to her primary care physician with a 15-month history of occasional nausea and vague abdominal discomfort, without associated pain, weight loss, or vomiting. Physical examination was normal, without palpable mass or tenderness. An ultrasound examination performed at another institution reportedly showed a pancreatic pseudocyst. She was subsequently referred to the University of Michigan Medical Center for further evaluation, where an upper GI series demonstrated a possible retrogastric mass. Because of the clinical suspicion of pancreatic disease, sonographic evaluation of the pancreas was requested and revealed a 2.5$\mathrm{cm}$ cystic mass arising from the proximal celiac trunk, diagnostic of a celiac artery aneurysm (Fig $1 \mathrm{~A}$ and $\mathrm{B}$ ). The body of the pancreas was well visualized and normal (Fig 1A). Subsequent angiographic confirmation was obtained preoperatively (Fig $1 \mathrm{C}$ and D), and surgical resection was performed with primary end-to-end anastomosis of the common hepatic artery to the proximal

From the Departments of Radiology and Surgery, University of Michigan Medical School, Ann Arbor. Manuscript received February 25, 1980; revised manuseript accepted May 12, 1980. For reprints contact Terry M. Silver, MD, Department of Radiology, University Hospital, Ann Arbor, MI 48109. splenic artery. No associated inflammatory tissue, pancreatic abnormality, or blood clot suggesting prior rupture was found. The patient experienced an uneventful postoperative course and was asymptomatic three months following aneurysmectomy.

\section{DISCUSSION}

Celiac artery aneurysms account for $4 \%$ of splanchnic aneurysmal disease, and a total of 81 cases has been reported in the world literature. ${ }^{2}$ The causes and clinical importance of celiac artery aneurysms have changed markedly in recent years. Most of the 58 aneurysms reported prior to 1950 were luetic in origin, and catastrophic rupture and death were exceedingly common. Although most of these patients were symptomatic, an antemortem diagnosis was established only once in this group of patients. Since 1950, 43 celiac artery aneurysms have been reported. In contrast to earlier reports, arteriosclerotic changes have been noted in most of these lesions. The most common clinical manifestation has been epigastric, abdominal, and back discomfort. Hemorrhage as a complication has been distinctly unusual in recent cases.

Recognition of celiac artery aneurysms during the past $30 \mathrm{yr}$ has occurred with increasing frequency with greater utilization of angiography to evaluate abdominal disease. The first patients to have the preoperative diagnosis confirmed were reported from our institution. ${ }^{3}$ Only 21 patients, including six from the University of Michigan, have been subjected to surgical intervention, all within the last $22 \mathrm{yr}^{1,4}$ Of these patients, $90 \%$ have had successful outcomes. This reflects not only improved vascular surgical techniques, but also, and more importantly, an ability to establish an earlier, accurate diagnosis. The difficulty in making a diagnosis is apparent since only 18 patients have had preoperative or antemortem recognition of their aneurysms. 

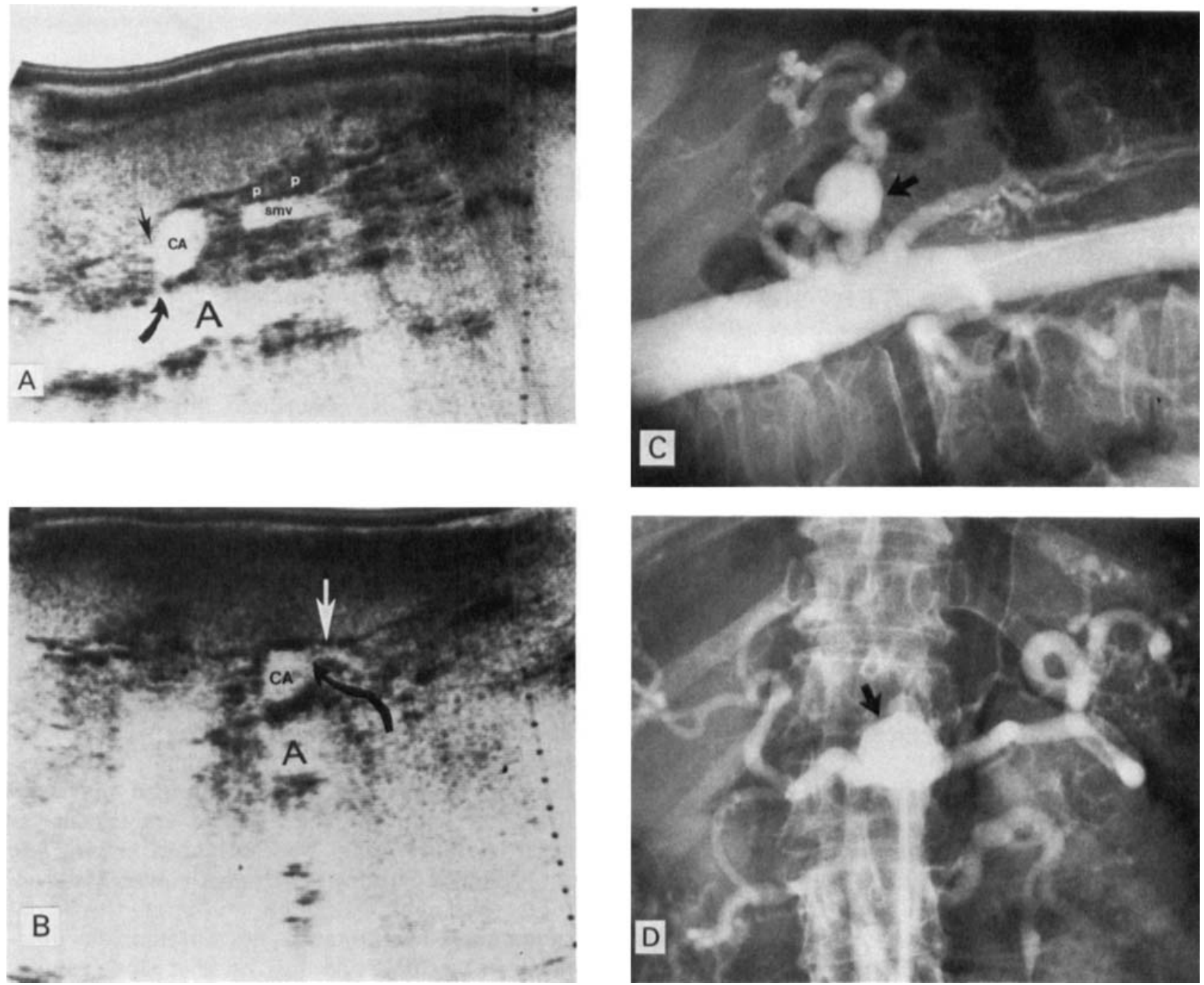

FIGURE 1. Celiac Artery Aneurysm. A: Sagittal midline scan showing celiac artery aneurysm (CA) arising from proximal celiac trunk (curved arrow). Note origin of left gastric artery (straight arrow) from aneursym. A: aorta. SMV: superior mesenteric vein. P: pancreas. B: Transverse scan 2 $\mathrm{cm}$ caudal to xiphoid process through celiac aneurysm (CA). Note origin (black arrow) and proximal portion (white arrow) of splenic artery, arising from celiac aneurysm. A: aorta. C: Lateral and D: anteroposterior (P) radiographs from aortogram showing celiac aneurysm (arrow).

With increasing utilization of sonography as a screening procedure for evaluating the upper abdomen, in particular the pancreas, asymptomatic pathologic masses are often encountered. Visualization of upper abdominal and retroperitoneal organs through recognition of reliable boundary landmarks, predominately vascular, is now routine..$^{5-8}$ Sample, ${ }^{5}$ emphasizing the importance of carefully evaluating these structures, described a cystic abdominal mass representing a hepatic artery aneurysm.

As demonstrated by our case, specific diagnosis of celiac artery aneurysm is possible if characteristic sonographic features are recognized (Fig $1 \mathrm{~A}$ and $\mathrm{B}$ ). The aneurysm appeared as a midline, spherical, cystic mass anterior to the aorta, just cephalad to the body of the pancreas, which was normal and separate from the aneurysm. Demonstration of continuity between the mass and splanchnic vessels, such as the proximal celiac trunk, left gastric, and splenic or hepatic arteries, establishes the correct diagnosis, as in this case. This requires meticulous scanning technique, including multiple sections obtained using various scanning angles and obliquities. High-resolution real-time scanning provides a valuable adjunctive, dynamic method for evaluating these lesions. The pulsatile nature of the mass and continuity with splanchnic vessels can be quickly established.

Since a celiac aneurysm will usually be discovered incidentally as a cystic, peripancreatic mass, awareness of the typical sonographic appearance permits correct differentiation from in- 
trinsic pancreatic masses, such as congenital cysts or pseudocysts. In addition, knowledge of the clinical and sonographic features of celiac aneurysms should lead to increased recognition of this entity. It is possible that the prevalence of splanchnic aneurysmal disease, including celiac aneurysms, is greater than currently clinically recognized.

\section{REFERENCES}

1. Stanley JC: Splanchnic Artery Aneurysms, in Rutherford RB (ed) Vascular Surgery. Philadelphia, W.B. Saunders \& Co, 1977, p 673.

2. Graham LM, Zelenock GB, Whitehouse W Jr, et al: Celiac artery aneurysm. (Submitted for publication.)
3. Kraft RO, Fry WJ: Aneurysms of the celiac artery. Surg Gynecol Obstet 117:563, 1963.

4. Haimovici H, Sprayregen S, Eckstein P, et al: Celiac artery aneurysmectomy: Case report with review of the literature. Surgery 79:592, 1976.

5. Sample WF: Techniques for improved delineation of normal anatomy of the upper abdomen and high retroperitoneum with gray-scale ultrasound. Radiology 124:197, 1977.

6. Filly RA, Carlsen EN: Newer ultrasonographic anatomy in the upper abdomen, II: The major systemic veins and arteries with a special note on localization of the pancreas. J Clin Ultrasound 4:91, 1976.

7. Leopold GR: Gray-scale ultrasonic angiography of the upper abdomen. Radiology 117:665, 1975.

8. Sample WF, Po JB, Gray RK, et al: Gray-scale ultrasonography: Techniques in pancreatic scanning. Appl Radiol 4:63, 1975. 